\title{
Geometric Mapping for Non-Rectangular Plates with Micro/Nano or Macro Scaled under Different Effects
}

\author{
Kadir Mercan ${ }^{a}, O_{m e r}$ Civalek $^{b^{*}}$ \\ ${ }^{\mathrm{a}, \mathrm{b}}$ Akdeniz University, Civil Engineering Dept., Antalya / TURKIYE \\ E-Mail address: kmercan@mehmetakif.edu.tr ${ }^{\text {a }}$, civalek@yahoo.com ${ }^{\text {b* }}$ \\ ORCID numbers of authors: \\ 0000-0003-3657-6274 $4^{a}$ 0000-0003-1907-9479
}

Received date: 10.10.2019

Accepted date: 08.11.2019

\begin{abstract}
The main purpose of this study is to give a perspective via discrete singular convolution, differential quadrature (DQ) and harmonic differential quadrature (HDQ). For this purpose, DQ and HDQ methods are developed for the buckling, analysis of non-rectangular plates. Plates of, skew, shape is considered under axial loads. Furthermore, transformation formulations and some perspective for nano or macro scaled many problems with different effects discussed via discrete singular convolution and differential quadrature methods.
\end{abstract}

Keywords: Discrete singular convolution, Harmonic differential quadrature; Plates; Graphene sheet, nonlocal elasticity, buckling.

\section{Introduction}

It is well known that, the analysis of engineering systems includes two main stages, such as; construction of a mathematical model for a given physical phenomena and the solution of this mathematical equation. Real physical systems or engineering problems are often described by partial differential equations, either linear or nonlinear and in most cases, their closed form solutions are extremely difficult to establish. As a result, approximate numerical methods have 
been widely used to solve partial differential equations that arise in almost all engineering disciplines. The most commonly used numerical methods for such applications are the finite element, finite difference, Ritz, and boundary element method, and most engineering problems can be solved by these methods to adequate accuracy if a proper and sufficient number of grid points are used. In addition to this, in a large number of practical applications where only reasonably accurate solutions at few specified physical coordinates are of interest, the conventional numerical methods such as finite element or finite difference method require a large number of grid points and so large a computer capacity. Among a variety of numerical methods, the finite element method is by far the most effectively and widely used method. Furthermore, finite element method is still an effective method in especially the systems with complex geometry and load conditions or applications with non-linear behavior and it has many successful applications. In seeking a more efficient numerical method that requires fewer grid points yet achieves acceptable accuracy, the method of differential quadrature (DQ) was introduced by Bellman et al. [1]. Since then, applications of differential quadrature method to various engineering problems have been investigated and their successes have demonstrated the potential of the method as an attractive numerical analysis technique [2-10]. The stability analysis of plates may be either closed form or approximate. The closed form solutions consist of techniques for seeking direct solutions to the governing differential equation of plates. A closed form or rigorous solution of plates can be obtained for only a limited number of cases. For the majority of practical problems, a closed form namely analytical solution either cannot be obtained or is of such a complicated nature that it can be applied only with great difficulty in a practical computation. For many situations, numerical methods are the only approaches that can be employed [11-17]. 
The focus of this paper is on the comparison study of the DQ and HDQ methods. For this reason, the applications are limited to those problems having smooth solutions for simplicity. In addition to this, since the only thin plates are considered in this paper, there are some assumptions regarding the behavior of thin plates. These are; the transverse deflections of the plates are small compared to the thickness of the plate. Thus middle-surface stretching caused by bending can be neglected; that is, membrane action resulting from flexure is negligible compared to the flexure. The material of the plate is homogeneous, isotropic, and obeys Hooke's law.

\section{Differential Quadrature Method (DQM)}

For simplicity, we consider a one-dimensional function $u(x)$ in the $[-1,1]$ domain, and $N$ discrete points. Then the first derivatives at point $i$, at $x=x_{i}$ is given by

$$
u_{x}\left(x_{i}\right)=\left.\frac{\partial u}{\partial x}\right|_{x=x_{i}}=\sum_{j=1}^{N} A_{i j} u\left(x_{j}\right) \quad ; i=1,2, \ldots \ldots . ., N
$$

where $x_{j}$ are the discrete points in the variable domain, $u\left(x_{j}\right)$ are the function values at these points and $A_{i j}$ are the weighting coefficients for the first order derivative attached to these function values. Bellman et al. [1,2] suggested two methods to determine the weighting coefficients. The first one is to let equation (1) be exact for the test functions

$$
u_{k}(x)=x^{k-1} ; k=1,2, \ldots, N
$$

which leads to a set of linear algebraic equations

$$
(k-1) x_{i}^{k-2}=\sum_{j=1}^{N} A_{i j} x_{j}^{k-1} ; \text { for } i=1,2, \ldots \ldots \ldots, N \quad \text { and } \quad k=1,2, \ldots \ldots \ldots, N
$$


which represents $N$ sets of $N$ linear algebraic equations. Thus, the weighting coefficients for each formula will be different from those for the first order derivative. As similar to the first order, the second order derivative can be written as

$$
u_{x x}\left(x_{i}\right)=\left.\frac{\partial^{2} u}{\partial x^{2}}\right|_{x=x_{i}}=\sum_{j=1}^{N} B_{i j} u\left(x_{j}\right) ; i=1,2, \ldots \ldots \ldots, N
$$

where the $B_{i j}$ is the weighting coefficients for the second order derivative.

Another way to determine the weighting coefficients is to employ harmonic functions, named the harmonic differential quadrature (HDQ). Harmonic differential quadrature has been proposed by Striz et al. [19]. Unlike the differential quadrature that uses the polynomial functions, such as power functions, Lagrange interpolated, and Legendre polynomials as the test functions, harmonic differential quadrature uses harmonic or trigonometric functions as the test functions. Thus, this method is called the HDQ method. Shu and Xue proposed an explicit means of obtaining the weighting coefficients for the HDQ [18]. The harmonic test function $h_{k}(x)$ used in this approach is defined as;

$$
\begin{gathered}
h_{k}(x)=\frac{\sin \frac{\left(x-x_{0}\right) \pi}{2} \cdots \cdots \cdot \sin \frac{\left(x-x_{k-1}\right) \pi}{2} \sin \frac{\left(x-x_{k+1}\right) \pi}{2} \cdots \cdot \sin \frac{\left(x-x_{N}\right) \pi}{2}}{\sin \frac{\left(x_{k}-x_{0}\right) \pi}{2} \cdots \cdots \cdot \sin \frac{\left(x_{k}-x_{k-1}\right) \pi}{2} \sin \frac{\left(x_{k}-x_{k+1}\right) \pi}{2} \cdots \cdot \sin \frac{\left(x_{k}-x_{N}\right) \pi}{2}} \\
\text { for } k=0,1,2, \ldots, N
\end{gathered}
$$

According to the HDQ, the weighting coefficients of the first-order derivatives $A_{i j}$ for $i \neq j$ can be obtained by using the following formula:

$$
A_{i j}=\frac{(\pi / 2) P\left(x_{i}\right)}{P\left(x_{j}\right) \sin \left[\left(x_{i}-x_{j}\right) / 2\right] \pi}, \quad i, j=1,2,3, \ldots \ldots . ., N
$$

where 


$$
P\left(x_{i}\right)=\prod_{j=1, j \neq i}^{N} \sin \left(\frac{x_{i}-x_{j}}{2} \pi\right), \quad \text { for } \mathrm{j}=1,2,3, \ldots \ldots . \mathrm{N}
$$

The weighting coefficients of the second-order derivatives $B_{i j}$ for $i \neq j$ can be obtained using following formula:

$$
B_{i j}=A_{i j}\left[2 A_{i i}^{(1)}-\pi c t g\left(\frac{x_{i}-x_{j}}{2}\right) \pi\right], \quad i, j=1,2,3, \ldots \ldots ., N
$$

\section{Applications of DQ methods}

The governing differential equations for skew plates under uniaxial compression (uniform normal force) $F_{x}$ along the $x$ direction and its differential quadrature form are given respectively,

$$
\begin{aligned}
& u_{x x x x}-(4 k \cos \theta) u_{x x x y}+2 k^{2}\left(1+2 \cos ^{2} \theta\right) u_{x x y y}-\left(4 k^{3} \cos \theta\right) u_{x y y y}+k^{4} u_{y y y y}= \\
& -\frac{a^{2}}{D} F_{x} \sin ^{4}(\theta / 4) u_{x} \\
& \sum_{n=1}^{N_{x}} D_{i n} u_{n j}-4 k \cos \theta \sum_{n=1}^{N_{x}} C_{i n} \sum_{m=1}^{N_{y}} A_{j m} u_{m n}+2 k^{2}\left(1+2 \cos ^{2} \theta\right) \sum_{n=1}^{N_{x}} B_{i n} \sum_{m=1}^{N_{y}} B_{j m} u_{i m} \\
& -2 k^{2}\left(1+2 \cos ^{2} \theta\right) \sum_{n=1}^{N_{x}} A_{i n} \sum_{m=1}^{N_{y}} C_{j m} u_{m n}+k^{2} \sum_{m=1}^{N_{y}} D_{j m} u_{m i}=\frac{a^{2}}{D} F_{x} \sin ^{4}(\theta / 4) \sum_{n=1}^{N_{x}} A_{i n} u_{m j} \\
& i=1,2, \ldots \ldots ., N_{x} \quad \text { and } \quad j=1,2, \ldots \ldots, N_{y}
\end{aligned}
$$

where $\theta$ is skew angle, $k=a / b$ is the aspect ratio, $u$ is the displacement in the $z$ direction, and $D$ is the flexural rigidity. Clamped support condition with movable edges is considered. In accordance with this condition the plate is prevented from moving in the $z$ direction or rotating at 
the boundaries. In this case both the deflection and slope must vanish. Since the first and last displacements are known, Eq. (36) is rewritten for boundary conditions

$$
\begin{aligned}
& \sum_{n=1}^{N_{x}} D_{i n} u_{n j}-4 k \cos \theta \sum_{n=1}^{N_{x}} C_{i n} \sum_{m=1}^{N_{y}} A_{j m} u_{m n}+2 k^{2}\left(1+2 \cos ^{2} \theta\right) \sum_{n=1}^{N_{x}} B_{i n} \sum_{m=1}^{N_{y}} B_{j m} u_{i m} \\
& -2 k^{2}\left(1+2 \cos ^{2} \theta\right) \sum_{n=1}^{N_{x}} A_{i n} \sum_{m=1}^{N_{y}} C_{j m} u_{m n}+k^{2} \sum_{m=1}^{N_{y}} D_{j m} u_{m i}=\frac{a^{2}}{D} F x \sin ^{4}(\theta / 4) \sum_{n=1}^{N_{x}} A_{i n} u_{m j} \\
& i=2, \ldots \ldots . ., N_{x}-1 \text { and } j=2, \ldots \ldots ., N_{y^{-}}-1
\end{aligned}
$$

Consequently, we solve the remaining eigenvalue problem to obtain the buckling loads.

\section{Discrete Singular Convolution (DSC)}

Wei [18-20] proposed the method of discrete singular convolution (DSC) in 1998. In this method, numerical solutions of differential equations are discrete via some kernels. A singular convolution defined below [19]

$$
F(t)=(T * \eta)(t)=\int_{-\infty}^{\infty} T(t-x) \eta(x) d x
$$

For example, regularized Shannon kernel (RSK) is more suitable for practical applications. This kernel can write as follows [18]

$$
\delta_{\Delta, \sigma}\left(x-x_{k}\right)=\frac{\sin \left[(\pi / \Delta)\left(x-x_{k}\right)\right]}{(\pi / \Delta)\left(x-x_{k}\right)} \exp \left[-\frac{\left(x-x_{k}\right)^{2}}{2 \sigma^{2}}\right] ; \sigma>0
$$


by using the method of DSC and DQ methods plates with different shape can be solve via below transformation rules

$$
\begin{gathered}
\frac{\partial^{2} w}{\partial x^{2}}=\left[J_{22}\right]^{-1} \sum_{i=-M}^{M} \delta^{(2)}{ }_{\Delta, \sigma}(k \Delta \xi) w_{i k}-\left[J_{22}\right]^{-1}\left[J_{21}\right]\left[J_{11}\right]^{-1} \sum_{i=-M}^{M} \delta^{(1)}{ }_{\Delta, \sigma}(k \Delta \xi) w_{i k} \\
\frac{\partial^{2} w}{\partial y^{2}}=\left[J_{22}\right]^{-1} \sum_{i=-M}^{M} \delta^{(2)}{ }_{\Delta, \sigma}(k \Delta \eta) w_{j k}-\left[J_{22}\right]^{-1}\left[J_{21}\right]\left[J_{11}\right]^{-1} \sum_{i=-M}^{M} \delta^{(1)}{ }_{\Delta, \sigma}(k \Delta \eta) w_{j k} \\
\frac{\partial^{2} w}{\partial x \partial y}=\left[J_{22}\right]^{-1} \sum_{i=-M}^{M} \delta^{(1)}{ }_{\Delta, \sigma}(k \Delta \xi) \sum_{i=-M}^{M} \delta^{(1)}{ }_{\Delta, \sigma}(k \Delta \eta) w_{i j} \\
-\left[J_{22}\right]^{-1}\left[J_{21}\right]\left[J_{11}\right]^{-1} \sum_{i=-M}^{M} \delta^{(1)} \Delta, \sigma(k \Delta \eta) w_{j k} \\
\Theta_{x}^{n}()=\frac{\partial^{(n)}()}{\partial x^{(n)}}=\sum_{k=-M}^{M} \delta_{\Delta, \sigma}^{(n)}(k \Delta x)()_{i+k, j} \\
\Theta_{y}^{n}()=\frac{\partial^{(n)}()}{\partial y^{(n)}}=\sum_{k=-M}^{M} \delta_{\Delta, \sigma}^{(n)}(k \Delta y)()_{i, j+k} \\
\Theta_{x}^{(n-1)} \Theta_{y}^{1}()=\frac{\partial^{(n)}()}{\partial x^{(n-1)} \partial y}=\sum_{k=-M}^{M} \delta_{\Delta, \sigma}^{(n-1)}(k \Delta x)()_{i+k, j} \sum_{k=-M}^{M} \delta_{\Delta, \sigma}^{(1)}(k \Delta y)()_{i, k+j} \\
\Theta_{x}^{1} \Theta_{y}^{(n-1)}()=\frac{\partial^{(n)}()}{\partial x \partial y^{(n-1)}}=\sum_{k=-M}^{M} \delta_{\Delta, \sigma}^{(1)}(k \Delta x)()_{i+k, j} \sum_{k=-M}^{M} \delta_{\Delta, \sigma}^{(n-1)}(k \Delta y)()_{i, k+j}
\end{gathered}
$$

Similar transformation can also possible for DQ or HDQ methods.

\section{Concluding remarks and nano-scaled plates}

In the applied mechanics area, many different problems can be solved via DSC or DQ transformation methods. For example, below cases can be consider for each plate problem (triangular, skew, trapezoidal, circular, annular, sector, polygonal, deltoid, or general nonrectangular plates:

5.1. Foundation effects, magnetic effects, piezo effect. 
5.2. Functionally graded (FG) composite material

5.3. CNT reinforced composite material

5.4. Graphene platelet reinforced composite material

5.5. Nonlinear analysis-material nonlinearity

5.6. Buckling

5.7. Bending

5.8. Vibration

5.9 Porosity effect

5.10. Post buckling

5.11. Nonlinear analysis-geometric nonlinearity

5.12. Viscoelasticity, damped vibration.

Also, micro/nano scaled plates, beam and shell problems can also be solved [21-31] via these methods.

\section{References}

[1] Bellman, R., Casti, J., Differential quadrature and long-term integration, Journal of Mathematical Analysis and Application, 34, 235-38, 1971.

[2] Bellman, R., Kashef, B.G., Casti, J., Differential Quadrature: A technique for the rapid solution of nonlinear partial differential equation, Journal of Computational Physics, 10, 40-52, 1972.

[3] Bert, C.W., Malik, M., The differential quadrature method for irregular domains and application to plate vibration, International Journal of Mechanical Science, 38(6), 589-606, 1996.

[4] Bert, C.W., Jang, S.K., Striz, A.G., Two new approximate methods for analyzing free vibration of structural components, AIAA Journal, 26(5), 612-18, 1987.

[5] Bert, C.W., Wang, Z., Striz, A.G., Differential quadrature for static and free vibration analysis of anisotropic plates, International Journal of Solids and Structure, 30(13),1737-44, 1993.

[6] Bert, C.W., Malik, M., Free vibration analysis of tapered rectangular plates by differential quadrature method: a semi- analytical approach, Journal of Sound and Vibration, 190(1), 4163, 1996.

[7] Bert, C.W., Wang, Z., Striz, A.G., Convergence of the DQ method in the analysis of an isotropic plates, Journal of Sound and Vibration, 170(1), 140-44, 1994.

[8] Bert, C.W., Malik, M., Differential quadrature method in computational mechanics: a review, Applied Mechanics Review, 49(1), 1-28, 1996. 
[9] Bert, C.W., Wang, Z., Striz, A.G., Static and free vibrational analysis of beams and plates by differential quadrature method, Acta Mechanica, 102, 11-24, 1994.

[10] Björck, A., Pereyra, V., Solution of Vandermonde system of equations, Mathematical computing, 24, 893-903, 1970.

[11] Civalek, Ö., Finite Element analysis of plates and shells, Elazı̆g: Firat University (in Turkish), 1998.

[12] Shu, C., Xue, H., Explicit computations of weighting coefficients in the harmonic differential quadrature, Journal of Sound and Vibration, 204(3), 549-55, 1997.

[13] Liew, K.M., Teo, T.M., Three dimensional vibration analysis of rectangular plates based on differential quadrature method, Journal of Sound and Vibration, 220(4), 577-99, 1999.

[14] Timoshenko, S.P., Gere, J.M., Theory Elastic Stability, McGraw-Hill, Second Edition, Tokyo, 1959.

[15] Chajes, A., Principles of Structural Stability Theory, Prentice-Hall, New Jersey, 1974.

[16] Wang, X., Striz, A.G., Bert, C.W., Buckling and vibration analysis of skew plates by the differential quadrature method, AIAA Journal, 32(4), 886-889, 1994.

[17] Wang, X., Bert, C.W., Striz, A.G., Differential quadrature analysis of deflection, buckling and free vibration of beams and rectangular plates, Computers and Structures, 48(3), 473-479, 1993.

[18] Wei, G.W., A new algorithm for solving some mechanical problems, Comput. Methods Appl. Mech. Eng., 190, 2017-2030, 2001.

[19] Wei, G.W., Vibration analysis by discrete singular convolution, Journal of Sound and Vibration, 244, 535-553, 2001.

[20] Wei, G.W., Discrete singular convolution for beam analysis, Engineering Structures, 23, 1045-1053, 2001.

[21] Akgöz, B., Civalek, O., Effects of thermal and shear deformation on vibration response of functionally graded thick composite microbeams, Composites Part B: Engineering 129, 77-87, 2017.

[22] Civalek, O., Geometrically non-linear static and dynamic analysis of plates and shells resting on elastic foundation by the method of polynomial differential quadrature (PDQ), Firat University, Elazı ̆̆ 2004.

[23] Civalek, O., Demir, C., Buckling and bending analyses of cantilever carbon nanotubes using the euler-bernoulli beam theory based on non-local continuum model, Asian Journal of Civil Engineering, 12(5), 651-661, 2011. 
[24] Akgoz, B., Civalek, O., Nonlinear vibration analysis of laminated plates resting on nonlinear two-parameters elastic foundations, Steel and Composite Structures, 11(5), 403-421, 2011.

[25] Civalek, O., Acar, M.H., Discrete singular convolution method for the analysis of Mindlin plates on elastic foundations, International Journal of Pressure Vessels and Piping, 84(9), 527535, 2007.

[26] Civalek, O., Yavas, A., Large deflection static analysis of rectangular plates on two parameter elastic foundations, International journal of science and technology, 1(1), 43-50, 2006.

[27] Civalek, O., Kiracioglu, O., Free vibration analysis of Timoshenko beams by DSC method, International Journal for Numerical Methods in Biomedical Engineering, 26(12), 1890-1898, 2010 .

[28] Demir, C., Civalek, O., A new nonlocal FEM via Hermitian cubic shape functions for thermal vibration of nano beams surrounded by an elastic matrix, Composite Structures, 168, 872-884, 2017.

[29] Mercan, K., Demir, C., Civalek, O., Vibration analysis of FG cylindrical shells with powerlaw index using discrete singular convolution technique, Curved and Layered Structures, 3(1), 82-90, 2016.

[30] Demir, C., Civalek, O., On the analysis of microbeams, International Journal of Engineering Science, 121, 14-33, 2017.

[31] Civalek, O., Geometrically nonlinear dynamic and static analysis of shallow spherical shell resting on two-parameters elastic foundations, International Journal of Pressure Vessels and Piping, 113, 1-9, 2014. 